\title{
APLICATIVO DE OSTEOLOGÍA PARA LOS PROGRAMAS DE MEDICINA VETERINARIA Y ZOOTÉCNIA DE LA UNIVERSIDAD DE PAMPLONA
}

\section{OSTEOLOGY APPLICATION IN PROGRAMS OF VETERINARY MEDICINE AND ZOOTECNIA OF THE UNIVERSITY OF PAMPLONA}

\author{
Ing. Juan Gutiérrez, MSc. José Santiago. \\ MSc. Fernando Gómez, MSc. Xavier Jaramillo. \\ Universidad de Pamplona. \\ Ciudadela Universitaria, Pamplona, Norte de Santander, Tel.: (+577) 5685003. \\ E-mail: jcarlos891126@ hotmail.com, \\ \{josesantiago, fernando.gomez, xavier.jaramillo\} unipamplona.edu.co
}

\begin{abstract}
Resumen: En este trabajo se desarrolla un aplicativo donde los estudiantes y docentes puedan realizar consultas y memorizar algunas funciones de las estructuras anatómicas de las especies equina, bovina y canina. Esta basado en el uso de fotografías tomadas a las estructuras óseas, para una posterior reconstrucción en 3D. El software creado, permite observar, estudiar e identificar características importantes del sistema óseo de dichas especies.
\end{abstract}

Palabras clave: Software, osteología, anatomía, aplicativo, TIC.

\begin{abstract}
This paper presents an application where students and teachers can perform queries and memorize some functions of anatomical structures of the equine, bovine and canine species develops. It is based on the use of photographs taken at the osseous structures, for subsequent 3D reconstruction. The software created, you can observe, study and identify important features of the skeletal system of these species.
\end{abstract}

Keywords: Software, osteology, anatomy, application, TIC.

\section{INTRODUCCIÓN}

La presente investigación, parte de la necesidad de aprendizaje de la asignatura Anatomía Animal por parte de los estudiantes, como fundamento importante en el proceso y desarrollo de la Medicina Veterinaria para su estudio practico, en los primeros semestres, $\mathrm{y}$ asimismo en asignaturas del área de profundización profesional del programa. De igual manera, se tiene en cuenta, los inconvenientes que genera orientar esta asignatura usando técnicas tradicionales a base de formaldehido y otros agentes químicos utilizados como conservantes, que presentan inconvenientes relacionados con alto número de animales para sacrificar, uso de químicos con consecuencias en la salud humana como son: irritación de la nariz y garganta, dificultades respiratorias, bronquitis, sensibilización alérgica y su clasificación como carcinógeno humano; de igual forma se presentan efectos negativos en el medio ambiente en el proceso de eliminación de residuos.

Particularmente en el caso de la Universidad de Pamplona, los estudiantes de Medicina Veterinaria y Zootecnia, deben desplazarse durante su tiempo de trabajo independiente hasta el laboratorio de Anatomía ubicado en la Granja Experimental Villa Marina, la cual se encuentra a 49 kilómetros del campus principal. Lo anterior demanda tiempo en el desplazamiento, riesgos y dificultad en el transporte. Dadas estas circunstancias, el estudio 
realizado de manera independiente por parte del estudiante es muy limitado, lo cual conlleva a la alta tasa de mortalidad académica. Sin embargo, con la implementación de la herramienta de software, el estudiante tiene acceso a ella durante las 24 horas del día, complementando las prácticas desde cualquier sitio con acceso a internet, sin necesidad de desplazamiento al laboratorio, maximizando el tiempo del estudiante en el desarrollo de la asignatura, dándole la facilidad y la interacción con estructuras óseas reales.

\section{ANTECEDENTES}

La historia de la Medicina Veterinaria en el mundo se remonta a las primeras civilizaciones, asociada a la cría de animales con fines de proveer alimentos de origen animal, vestido, calzado y trabajo, luego para la guerra. Aunque la disciplina como tal, fue establecida hace 250 años (González, 2011).

De igual manera, en relación a la forma que se han conservado los cuerpos a través de la historia, sobresalen algunas técnicas a base de químicos como soluciones de formaldehido en diferentes concentraciones del 10\% al 15\% (Muñetón, 2013).

En el siglo XVIII las técnicas de conservación experimentaron un importante desarrollo debido principalmente a los siguientes investigadores (Beltran, 2009):

- Guillermo Hunter (1718-1783) utilizó el alcohol como medio de fijación y conservación.

- Pierre Dionis (1643-1718) empleó el ácido tánico con el fin de evitar el crecimiento de hongos.

- François Chaussier (1746-1828) se sirvió del sublimado o bicloruro de mercurio para evitar la putrefacción y favorecer la momificación.

- Johann Jacob Ritter (1714-1784) utilizó el arsénico.

- Karl Wilhelm Scheele (1742-1786) aplicó la glicerina para la conservación de cadáveres.

Más tarde en 1859, se dio el descubrimiento del formaldehído por parte del científico alemán William Hoffman. Este es un gas de olor penetrante, soluble en agua, con poderosas acciones conservantes ya que tiene un amplio espectro microbicida. Desafortunadamente se le reconoce como probable carcinógeno humano, por lo cual fue incluido por parte del Instituto Nacional de Cancerología en dicha lista (Muñetón C. \&., 2012).

A la fecha, en las salas de disección se utiliza el formol como medio químico básico de las innumerables fórmulas de conservación de cadáveres y piezas anatómicas, asociado a otras sustancias como: la glicerina, el alcohol, el fenol, el timol, el arsénico, el cloruro de sodio, el cloruro de zinc, el sulfato de potasio, el hidrato de cloral, el ácido acético, el bicarbonato de sodio, por citar los más importantes. A lo anterior se añaden otras técnicas de conservación con excelentes resultados, entre ellas se debe mencionar al profesor Gunther Von Hagens con su técnica de plastinación con base en el empleo de la acetona y la silicona (Heildelberg, 1989).

Dadas las dificultades relacionadas con los efectos secundarios y los costos de la preservación química, en las aulas de clase se complementa la enseñanza mediante el uso de entornos virtuales de aprendizaje. Para el ello se recurre a las TIC, como herramienta importante en los procesos académicos del siglo XXI. Es habitual hablar de este tipo de cambios propiciado por los avances de las tecnologías, como del inicio de una nueva era, a la que suele llamarse sociedad de la información. Se pueden destacar cuatro importantes temas que convergen en este momento (Duderstand, 1997):

- La importancia del conocimiento como un factor clave para determinar seguridad, prosperidad y calidad de vida.

- La naturaleza global de la sociedad.

- La facilidad con la que la tecnología de los ordenadores, las telecomunicaciones y la multimedia posibilitan el rápido intercambio de información.

- El grado con el que la colaboración informal (sobre todo a través de redes) entre individuos e instituciones está reemplazando a estructuras sociales más formales, como corporaciones, gobiernos, entre otros.

De igual manera, como lo afirma (Salinas, 2004), "para adaptarse a las necesidades de la sociedad actual, las instituciones de educación superior deben flexibilizarse y desarrollar vías de integración de las tecnologías de la información y la comunicación en los procesos de formación". El mismo autor manifiesta que se debe promover "experiencias innovadoras" en los procesos de enseñanza-aprendizaje, apoyándose en las TIC y haciendo énfasis en la docencia, en los cambios de estrategias didácticas de los profesores y en los sistemas de comunicación y distribución de los materiales de aprendizaje; es decir, en los procesos de innovación docente.

Para tales efectos, (Cortina, 2001) cita dos experiencias pioneras del cambio en instituciones 
de educación superior clásicas: la del MIT (Massachussets Institute of Technology y la del NIJT (New Jersey Institute of Technology), siendo ambas experiencias también ejemplares por su capacidad de uso de las TIC como recursos docentes y de visibilidad para la propia institución. Añade la autora que las TIC servirán para cambiar los esquemas clásicos de la enseñanza basados en la acumulación de conocimiento. La plataforma de software libre más extendida en el contexto universitario es Moodle, la cual se ha venido implementado paulatinamente en los ambientes universitarios (Prendes, 2011).

En el contexto local, en la Universidad de Pamplona el proceso de virtualización ha avanzado a grandes pasos, ello a juzgar por el desarrollo de sistemas de información que tecnifican los procesos administrativos y académicos. Aunque cabe resaltar que en éste último hace falta abarcar variados procesos relacionado con la docencia.

\subsection{Herramientas y Metodología}

El trabajo se basó en una investigación de campo aplicada, que para el caso en particular partió de la adecuación de los huesos en su estado natural, para posteriormente proceder a la toma de fotografías de alta resolución. Se tomó como muestra los sistemas óseos de ciertos animales, indispensables para el estudio veterinario y zootécnico como son los equinos, bovinos y caninos.

Para abordar con mayor grado de detalle el tema estudiado, y a su vez determinar los procesos a desarrollar dentro de la investigación, se hizo uso de instrumentos y fuentes de consulta entre los que sobresale la ejecución de entrevistas no estructuradas, tendientes a recabar las opiniones y conocimientos por parte de expertos en el área de desarrollo de software, al igual que en lo relacionado a la anatomía animal. Por otra parte, se desarrollaron procesos de consulta bibliográfica en bases de datos disponibles, tales como: Web of Science, Scopus, Proquest, Redalyc con el objetivo de determinar el estado del arte en proyectos similares.

Dentro de las principales actividades desarrolladas durante la presente investigación, en la figura 1 se esboza a grandes rasgos las fases del proyecto. En un primer momento se documenta el tema de estudio, en lo relacionado a los desarrollos tecnológicos, así mismo los aspectos relacionados con la anatomía animal y sus procesos de conservación. Posteriormente se presentan las fases de diseño, toma de fotografías, desarrollo del aplicativo y por último la validación y puesta en marcha del desarrollo.

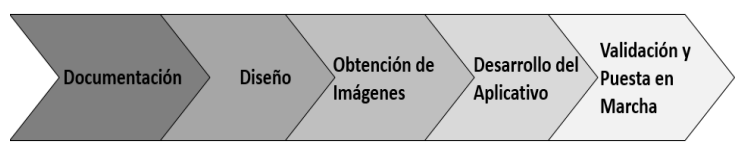

Fig. 1. Fases del proyecto.

Fase I. Objetivo: documentar el estado del arte para el estudio óseo de los equinos y cotejo respecto a otros sistemas óseos.

Fase II. Objetivo: diseñar un aplicativo de software para el estudio de la Osteología Veterinaria.

Fase III. Objetivo: realizar una base de datos para el acceso de las imágenes y posterior utilización de la misma para controlar las imágenes empleadas por el aplicativo.

Fase IV. Objetivo: implementar el aplicativo para el estudio de la osteología equina.

Fase V. Objetivo: Validar estructuralmente y conceptualmente el contenido del aplicativo por un especialista.

Es importante destacar que en el desarrollo del aplicativo, se hizo uso de herramientas de software, tales como NetBeans, Dreamweaver, Flash CS5 entre otros.

A través de la plataforma NetBeans se crearon los archivos Java, su uso es importante dado que las aplicaciones construidas a partir de módulos pueden ser extendidas agregándole nuevos módulos. Debido a que los módulos pueden ser desarrollados independientemente, las aplicaciones basadas en la plataforma NetBeans pueden ser extendidas fácilmente por otros desarrolladores.

Por otra parte, para darle mayor grado de diseño al aplicativo, se hizo uso de Dreamweaver CS5, ya que éste software permite la creación de páginas web dinámicas de alta calidad. Este programa permite desarrollar aplicaciones en varios lenguajes de programación, tales como: HTML, PHP, CSS, XML, JavaScript, entre otros. De igual forma, con el objetivo de dinamizar el entorno del aplicativo, se recurrió al uso del software de animación Flash CS5 bajo entornos de trabajos Action Script 3.0. Este software en particular, permite el desarrollo de animaciones en la construcción de elementos interactivos.

Para efectos de tomar las fotografías, se usó una cámara profesional Canon 5600 con un foco de 18 $\mathrm{mm}$ la cual permitió el logro de características 
apropiadas de resolución, enfoque, contraste y brillo en las imágenes. Posterior a la toma de las imágenes y su respectivo procesamiento y adecuación en el aplicativo, al final se obtiene un aplicativo ejecutable (.exe), el cual se desarrolló mediante el uso de un compilador $\mathrm{C}++$ denominado HM NIS versión 2.0.

\section{RESULTADOS}

Una vez finalizada la etapa de desarrollo y realizados los procesos de prueba, se pone en marcha el aplicativo. En lo relacionado a su funcionalidad, cabe resaltar la importancia de la portabilidad, ya que éste se puede acceder desde cualquier lugar donde exista conexión a internet. Una vez el estudiante o docente acceda al aplicativo, obtiene un entorno visual similar al presentado en la figura 2.

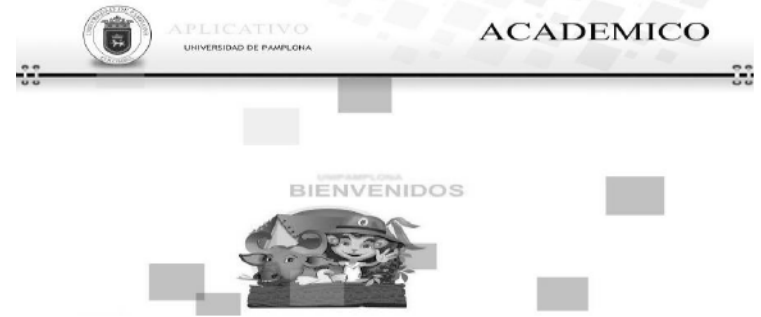

Fig. 2. Aplicativo de Osteología para el estudio de la Anatomía Animal.

\subsection{Módulos}

El aplicativo se compone de dos módulos generales: el primero corresponde al menú principal, en el cual el estudiante puede acceder y elegir la especie a estudiar, al igual que el hueso en particular a mostrar de dicha especie elegida.

Igualmente, existe un segundo módulo relacionado con el proceso de evaluación, en el cual el estudiante puede evaluar sus conocimientos mediante el desarrollo de un test (Ver figura 3).

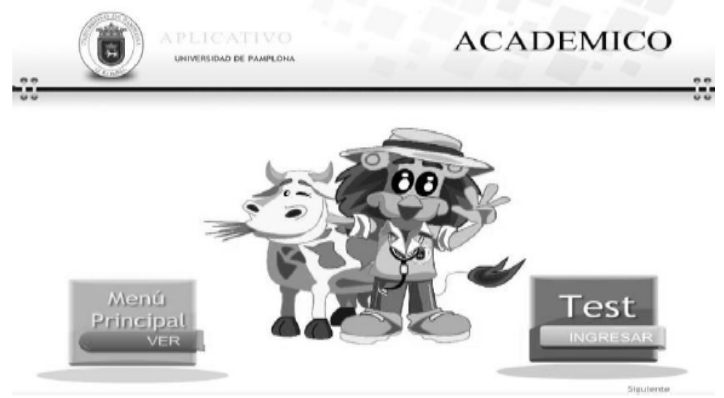

Fig. 3. Módulos principales del Aplicativo.

\subsubsection{Módulo Menú General}

En este módulo, el usuario encuentra un entorno visual que le permite navegar por otros submódulos correspondientes al visor de 3D, el menú registro, el contacto con el docente y de igual manera al módulo principal de evolución (test) que se menciona anteriormente (Ver figura 4).

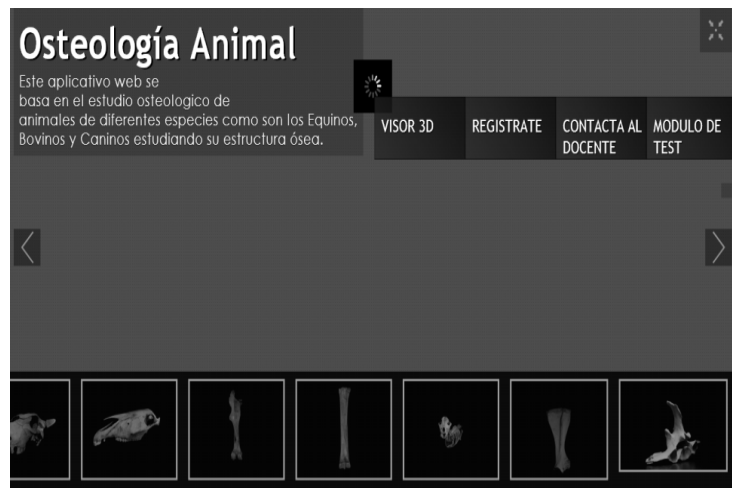

Fig. 4. Panel de opciones para acceder a submódulos.

Una vez el usuario elige el sub-módulo 3D, puede seguidamente elegir las diferentes especies y a su vez los componentes (huesos) que la componen, tal cual como se observa en la figura 5. Dicho visor brinda la posibilidad de rotar el hueso y a su vez realizar zoom para la ampliación de los accidentes del mismo.

\subsubsection{Módulo de Evaluación}

Una vez el usuario ingresa al módulo denominado test, puede evaluar los conocimientos adquiridos a través del aplicativo. Dicho test se alimenta de un banco de preguntas que el docente previamente debe registrar. De igual forma, dicho usuario debe haberse registrado previamente para acceder a la plataforma.

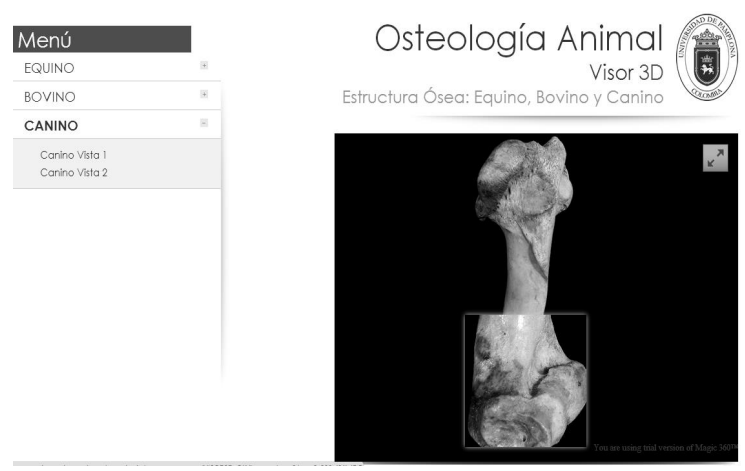

Fig. 5. Visor 3D. 


\section{CONCLUSIONES}

En términos generales, las bondades del presente trabajo, están encaminados a mejorar los niveles de asimilación de conocimientos por parte de los estudiantes de la asignatura Anatomía Animal de los Programas Medicina Veterinaria y Zootecnia de la Universidad de Pamplona. De igual manera cabe indicar, que la medición y análisis de resultados de momento hacen parte de una investigación en desarrollo y que por lo tanto no se encuentran dentro de los alcances de este artículo.

Por otro lado, es importante consignar el alto grado de aceptación por parte del Panel de expertos indagados, pertenecientes a las áreas de Ciencias Agrarias e Ingeniería. Dichos expertos manifestaron y resaltaron el gran potencial y el nivel de diseño implementado en el aplicativo. El proyecto ejecutado, es la plataforma base el desarrollo de otros trabajos proyectados dentro de la alianza entre la Facultad de Ingeniería y la Facultad de Ciencias Agrarias de la Universidad de Pamplona; entre ellos se destacan: aplicativos interactivos de Osteología en otras especies, aplicativos de Miología, Sistemas de Control Automáticos para Alimentación de animales domésticos, Sistemas de Medición Electrónicos, entre otros

\section{RECONOCIMIENTOS}

Este trabajo ha sido desarrollado en colaboración de los grupos de Investigación GICA y CICOM adscritos a la Vicerrectoría de Investigaciones, que a su vez pertenecen a la facultad de Ciencias Agrarias y a la Facultad de Ingenierías y Arquitectura de la Universidad de Pamplona. De igual manera, este Proyecto forma parte de la Tesis "Desarrollo de un Aplicativo de Osteología para el Programa de Medicina Veterinaria y Zootecnia de la Universidad de Pamplona" realizada por el egresado Juan Carlos Gutiérrez del Programa Ingeniería en Telecomunicaciones, el cual obtuvo Mención Meritoria.

\section{REFERENCIAS}

Beltran, J. (2009). Historia de la preservación de cadáveres. Morfología, pp 5-10.

Cortina, C. M. (2001). Las nuevas tecnologías, oportunidad y reto para la educación superior. Boletín de Educación Superior. CRUE.

Duderstand, J. (1997). The future of the university in an age of knowledge. Journal of Asynchronous.

González, S. C. (2011). Los 250 años de los estudios de las ciencias veterinarias. Mundo Pecuario, pp 151-166.

Heildelberg, H. V. (1989). Plastination Folder. Heildelberg: Anatomisches Institut I. Universitat Heildelberg.

Muñetón, C. \&. (2012). Plastinación: un instrumento complementario para el desarrollo del proceso enseñanza-aprendizaje de la anatomía. Rev Médica Veterinaria, pp 112-118.

Muñetón, G. A. (2013). Preparación en glicerina: una técnica para la conservación prolongada de cuerpos en anatomía veterinaria. Rev Médica Veterinaria, pp 115-122.

Prendes, E. P. (2011). Innovación con TIC en enseñanza superior: descripción y resultados de experiencias en la Universidad de Murcia. REIFOP, pp. 267-280.

Salinas, J. (2004). Innovación docente y uso de las TIC en la enseñanza universitaria. . Rev. Universidad y Sociedad del Conocimiento, pp 1-16. 